\title{
Magnetic mapping of hercynite produced by combustion synthesis
}

Jesana Moura $^{1}$, Renan Loreto ${ }^{2}$, Jefferson F. D. F de Araujo ${ }^{3}$ and Guillermo Solórzano ${ }^{4}$

${ }^{1}$ Department of Chemistry - PUC-Rio, Rio de Janeiro - RJ- Brazil - 22430-060, United States, ${ }^{2}$ Brazilian Center for Physical Research (CBPF), Rio de Janeiro - RJ- Brazil - 22290-180, United States, ${ }^{3}$ Departamento de Física, PontifíciaUniversidadeCatólica do Rio de Janeiro, Rio de Janeiro, Rio de Janeiro, Brazil, ${ }^{4}$ Department of Chemical and Materials Engineering - PUC-Rio, Rio de Janeiro - RJ- Brazil - 22430-060, Rio de Janeiro, Rio de Janeiro, Brazil

Spinel-type ferrites have attracted great attention due to its structural characteristics and potential applications, specially related to their magnetic properties. Spinel structured materials have FCC structure and general formula $\mathrm{AB} 2 \mathrm{O} 4$ [1] and can be synthetized by several methods, including co-precipitation, sol-gel [2] and combustion reaction [3]. The latter is particularly convenient, even for nanoparticles, due the low cost, high reaction speed, that may take only a few minutes, and for being energetically efficient, because the reaction environment releases enough energy for the reaction to occur [4]. In this sense, we have synthetized and characterized hercynite particles (FeAl2O4 - iron-aluminum spinel) with the method described in references $[1,3]$, with citric acid as a combustible. A solution with the iron and aluminum nitrates (in the molar ratio of 1:2) and citric acid, in a stoichiometric proportion, was mixed in a glass evaporation dish. The solution was heated in a hot plate at $500^{\circ} \mathrm{C}$, inside a fume hood until ignition and burning, to produce the solid product in powder. X-ray Diffraction (XRD), Scanning Transmission Electron Microscopy (STEM - JEOL2100F) and Scanning Electron Microscopy (SEM - JEOL7100F) was used to characterize this material.

Figure 1a shows the X-ray diffractogram of this sample, corresponding to a multiphasic material, with the typical structure of FeAl2O4 spinel (JCPDF 01-089-1685) and hematite (JCPDF 00-013-0534). Figures 1b and 1c are secondary electron (SE) and backscattered electron (BSE) images. Those micrographs indicate that this material have a morphology of solid aggregates with two phases, corroborating with figure 1a. Figures 1c and 1d are bright-field (BF) and dark-field (DF) STEM images, confirming the crystalline aggregate nature of this product, with particle size of around $40-50 \mathrm{~nm}$.

Magnetic properties were measured using a scanning magnetic microscope. Through a method of obtaining magnetic maps it's possible to obtain the region of maximum intensity as shown in figure $2 \mathrm{a}$. After this process we use the line method to obtain the magnetic moment of the sample [5]. This method can also be used through magnetic maps in two dimensions on the $\mathrm{X}$ and $\mathrm{Y}$ axis (See figure $2 \mathrm{~b}$ ). 
Acknowledgments

The authors thank LabNano (CBPF) for infrastructure. CNPq, Capes and Faperj for financial support.
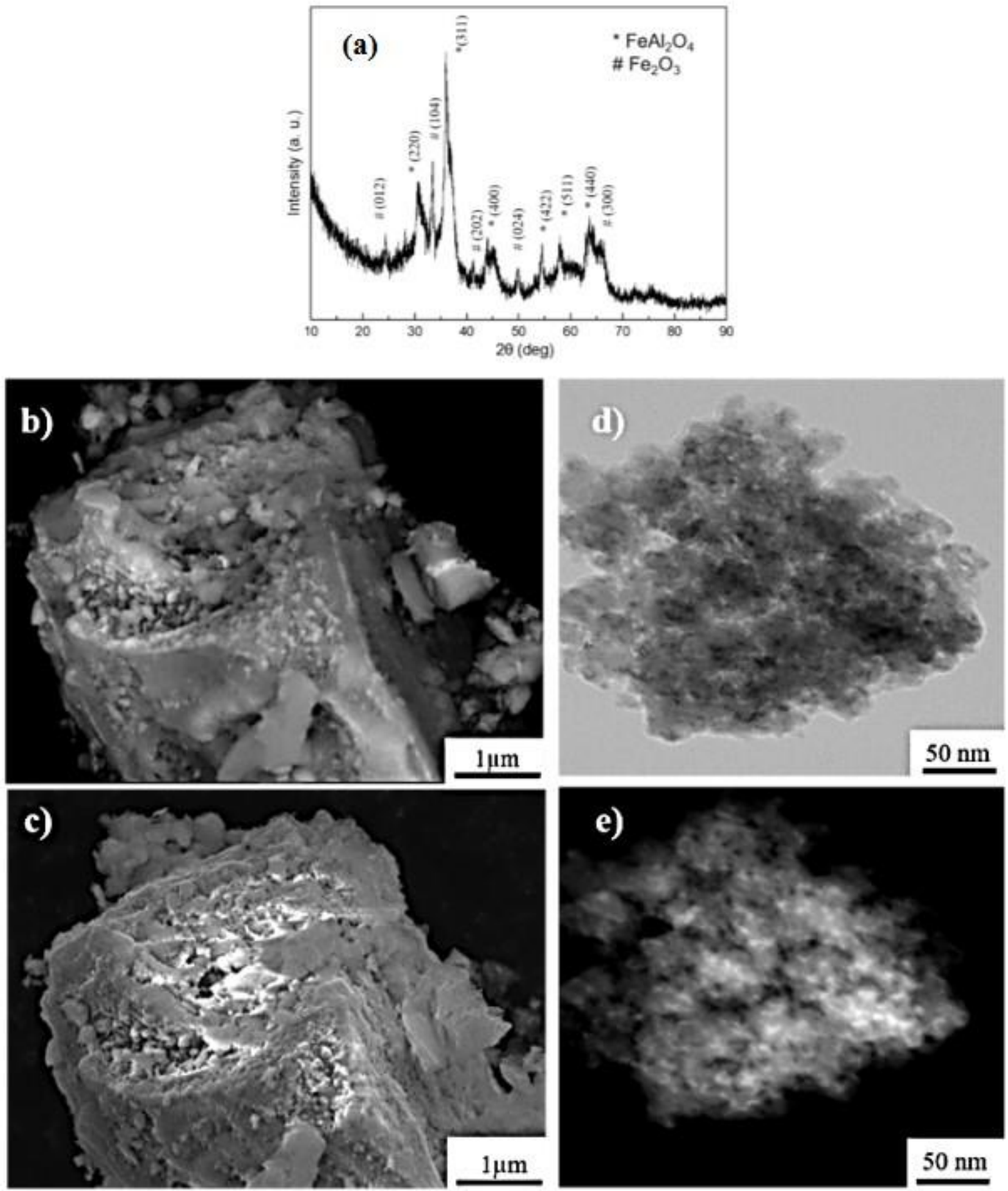
Figure 1. (a) X-ray diffractogram of the synthesis final product; (b) SE-SEM (c) BSE-SEM and (d) (e) STEM images, respectively

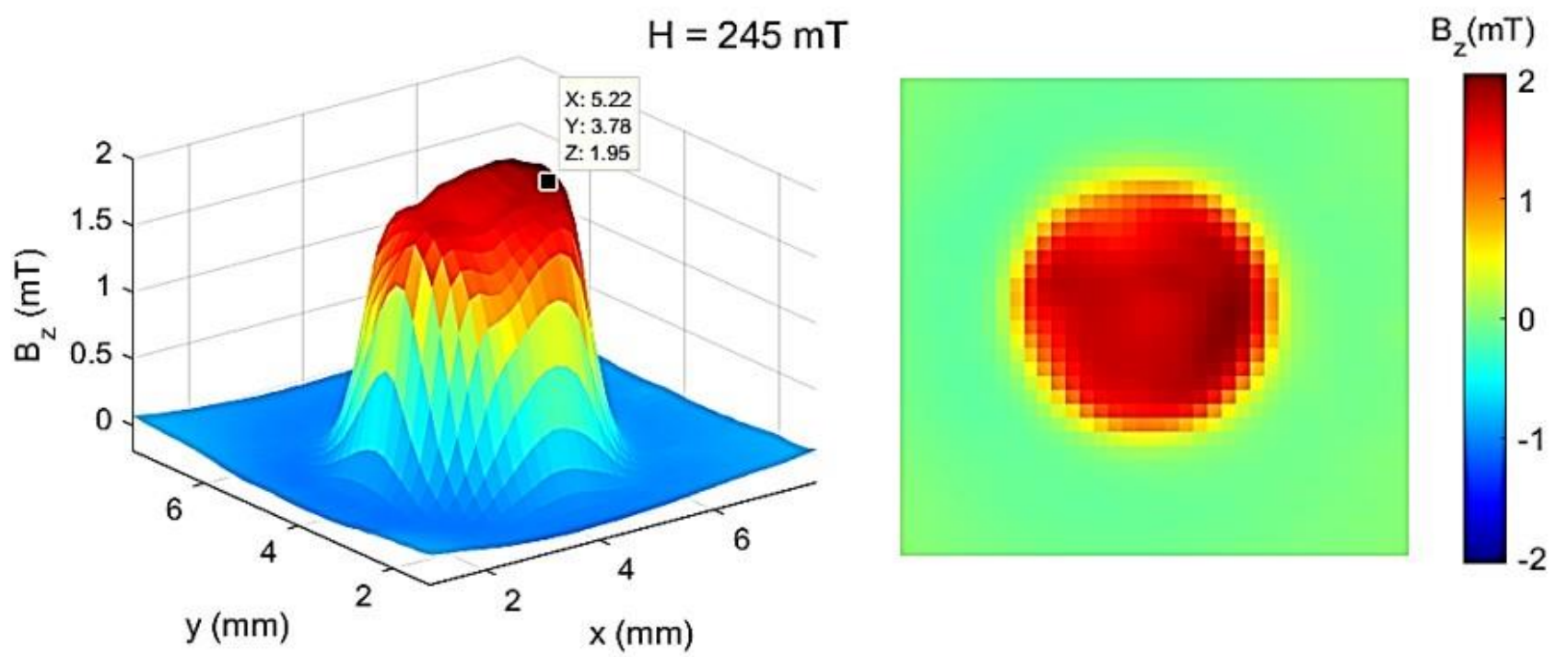

Figure 2. (a) Magnetic mapping of hercynite particles in 3 dimensions; (b) Magnetic mapping of hercynite particles in 2 dimensions.

\section{References}

[1] Silva, J. M., Araujo, J. F. D. F., Brocchi, E., \&Solorzano, I. G. (2020). Ceramics International, 46(11), 19052-19061.

[2] Millican, S. L., Androshchuk, I., Tran, J. T., Trottier, R. M., Bayon, A., Al Salik, Y., \& Weimer, A. W. (2020). Chemical Engineering Journal, 401, 126015.

[3] Loreto, R., Silva, J., Araujo, J., \& Solórzano, G. (2020). Microscopy and Microanalysis, 26(S2), 21542155.

[4] M. Chai et al. Chemistry Letters 43 (2014), 1743-1745.

[5] J. F. D. F. Araujo et al., J. Magn. Magn. Mater. 499 (2020), 166300 